\section{Cahiers de Narratologie}

Analyse et théorie narratives

8 | 1997

Création de l'espace et narration littéraire

\title{
Espace et lecture : la fonction des lieux dans la construction du sens
}

Vincent Jouve

\section{(2) OpenEdition}

1 Journals

Édition électronique

URL : http://journals.openedition.org/narratologie/10757

DOI : $10.4000 /$ narratologie. 10757

ISSN : 1765-307X

Éditeur

LIRCES

\section{Édition imprimée}

Date de publication : 1 décembre 1997

Pagination : 177-191

ISBN : 291089746X

ISSN : $0993-8516$

\section{Référence électronique}

Vincent Jouve, «Espace et lecture : la fonction des lieux dans la construction du sens », Cahiers de Narratologie [En ligne], 8| 1997, mis en ligne le 01 décembre 2020, consulté le 25 février 2021. URL http://journals.openedition.org/narratologie/10757 ; DOI : https://doi.org/10.4000/narratologie.10757

Ce document a été généré automatiquement le 25 février 2021.

Article L.111-1 du Code de la propriété intellectuelle. 


\title{
Espace et lecture : la fonction des lieux dans la construction du sens
}

\author{
Vincent Jouve
}

1 Parmi les développements les plus récents de la théorie littéraire, la réflexion sur la lecture occupe une place importante. En liant les problèmes de poétique (comment le texte est-il fabriqué?) aux problèmes de réception (en vue de quel effet est-il construit?), les analyses de la lecture ont permis d'associer la question du pourquoi à celle du comment. Elles apparaissent ainsi non comme la négation, mais comme le prolongement des études poéticiennes.

2 L'objectif de cette communication est d'examiner de quelle manière et à quels niveaux l'espace se donne à lire. Ce que je voudrais montrer, c'est que la représentation de l'espace au sein de la fiction conditionne toutes les composantes de l'acte de lecture.

3 Mais qu'entend-on exactement par «lecture »? Nombreux sont les modèles qui ont tenté de rendre compte de la complexité de la pratique lectorale. L'un des plus complets est sans doute celui de Gilles Thérien ${ }^{1}$. Outre l'aspect neurophysiologique qui ne nous intéresse pas ici, l'acte de lire comprend, selon lui, quatre dimensions :

- cognitive (la lecture est d'abord un travail de compréhension et de représentation);

- affective (l'attrait de la lecture tient en grande partie aux sentiments qu'elle suscite, aux jeux d'identification qu'elle propose);

- argumentative (le texte, résultat d'une volonté créatrice, ensemble organisé d'éléments, est toujours analysable comme discours, prise de position sur le monde et les êtres);

- symbolique (qu'elle les récuse ou les conforte, toute lecture interagit avec les schémas dominants d'une époque).

Le traitement textuel de l'espace informe chacune de ces dimensions. Pour le montrer, j'envisagerai successivement les quatre questions suivantes : espace et représentation, espace et identification, espace et intention, espace et signification. 


\section{Espace et représentation : le rôle des lieux dans l'activité cognitive}

5 Sur le plan cognitif, les lieux décrits dans le texte déterminent le travail de représentation du lecteur et le choix de son régime de lecture.

\section{Le cadre et la représentation de l'action}

6 Lire un texte, c'est d'abord comprendre ce qui s'y passe. La première tâche du lecteur consiste à identifier les événements relatés par le récit. Or, comme l'a montré Bertrand Gervais $^{2}$, la façon dont le lecteur se représente une action est directement liée au cadre dans lequel elle se déroule.

7 Le lecteur, pour passer d'une scène à l'autre, a en effet besoin de percevoir le lien qui les unit. Camper un décor stable au sein duquel varient événements et personnages est un moyen efficace de créer ce lien. La permanence du cadre permet de rassembler des faits épars en leur donnant une logique qui les rend lisibles. Tous les récits ayant pour titre un nom de lieu jouent consciemment de ce principe : que l'on pense à des textes comme Le Château des Carpates, Les Hauts de Hurlevent ou Le Pont de la rivière Kwaï. Mais d'autres romans, dont le titre ne comprend a priori aucun sème spatial, exploitent également ce procédé. Ainsi, dans La Vie Mode d'emploi de Perec, c'est la permanence du lieu qui permet de relier les événements divers qui se déroulent au 11, rue SimonCrubellier. Si certains habitants participent d'une histoire commune (le milliardaire Bartlebooth commande des puzzles à l'artisan Winckler à partir des toiles qu'il a réalisées grâce aux leçons du peintre Valène), c'est parfois le seul fait d'habiter le même immeuble qui autorise à rapprocher des personnages dont les trajectoires ne se rencontrent jamais. Dans un autre registre, on peut penser à la ville de Shangaï dans $L a$ Condition Humaine. Le roman de Malraux présente plusieurs intrigues qui ne s'entrecroisent que dans la mesure où elles se déroulent dans le même espace. Si le terroriste Tchen et l'idéaliste Kyo sont tous deux membres du parti communiste, rien, si ce n'est leur présence commune à Shangaï, ne destinait a priori à se rencontrer l'amateur d'art Clappique, le capitaine d'industrie Ferral et l'opiomane Gisors. Un cas intéressant est celui de la ville de Bleston dans L'Emploi du temps de Butor où l'unité de lieu contrebalance la complexité de la structure temporelle. Le récit narre le séjour d'un jeune français dans une ville anglaise où il effectue un stage d'un an. Entreprenant, après plusieurs mois, de raconter ce qui lui arrive en commençant par le début, le personnage-narrateur nous propose un journal où se mêlent passé et présent, récit et discours, dans un va-et-vient temporel qui n'hésite pas à remonter au passé mythique de la fondation de la ville. L'espace blestonien devient dès lors, en dépit de sa dimension labyrinthique, le seul repère stable pour le lecteur.

8 Si la référence à l'espace facilite la représentation de l'action, c'est aussi grâce à la mise en place de scénarios prédéterminés ou "scripts " ${ }^{3}$. Les "scripts de situation" (enchaînements de faits fondés sur le cadre) permettent cette intersection entre le monde du texte et le monde du lecteur sans laquelle il n'est pas de lecture possible. Tout cadre en effet définit un déroulement d'actions prévisible et clos, donc immédiatement reconnaissable par le lecteur. Des lieux aussi différents qu'un sousmarin (Vingt mille lieues sous les mers), un manoir isolé (Le tour d'écrou) ou une mine de charbon (Germinal) n'impliquent pas les mêmes possibles narratifs. La mention par le 
texte de tel ou tel lieu délimite automatiquement pour le lecteur une frontière entre ce qui peut et ce qui ne peut pas s'y passer. Parmi les «scripts de situation ", il faut faire une place particulière à ce que B. Gervais appelle les "scipts d'usage ", enchaînements d'actions fondés sur les rôles et accessoires propres à un cadre donné (dans un restaurant, on s'attend à trouver des serveurs et des clients, des assiettes et des couverts; dans une mine, des contremaîtres et des mineurs, des pioches et des wagonnets, etc.).

9 Mais le récit peut aussi choisir d'inventer pour tel espace un script inédit qui, une fois établi, facilitera, en se répétant, le travail de lecture (ainsi, dans l'œuvre de Sade, la mention du château isolé appelle le script «orgie» qui ne lui est pas, a priori, spontanément associé).

10 Cette exploitation des scripts liés à l'espace est essentielle: fondant l'adhésion du lecteur, elle est le «je sais que » qui libère pour le «que va-t-il se passer?» (c'est, insistons-y, le savoir commun qui permet l'intersection entre le monde du texte et le monde du lecteur, la lisibilité étant fondée sur la reconnaissance ${ }^{4}$ ).

11 Les deux types de script (scripts inédits et scripts d'usage) ont cependant leurs limites. Un texte fondé sur un savoir déjà partagé a peu de choses à dire, Ainsi, le paysage champêtre éclairé par la lune et bercé par le chant des grillons appelle-t-il traditionnellement une scène d'effusion sentimentale, script tourné en dérision par Flaubert dans Madame Bovary ${ }^{5}$. La surutilisation des scripts d'usage est à la base de la stéréotypie ${ }^{6}$. Mais, à l'inverse, si l'invention de scripts inédits garantit en général l'intérêt d'un récit, un script trop original peut souffrir d'une programmation insuffisante (le lecteur est alors incapable de reconstituer les déroulements d'actions en jeu). C'est ce qui se produit à la lecture de certains romans de science-fiction qui nous confrontent à des espaces par trop inédits. Cet effet peut cependant être volontaire quand la visée du texte est ouvertement de confronter le lecteur à un monde déconcertant (comme le fait Lewis Caroll dans Alice au pays des merveilles). Dans Le Viceconsul, c'est pour signifier l'instabilité des frontières entre les univers objectif et subjectif que Duras fait le choix d'une programmation insuffisante. Ainsi, à la fin du roman, le brusque passage d'un personnage à l'autre rend particulièrement difficile la représentation de ce qui se passe dans la chambre de l'hôtel, The Prince of Wales, où évoluent Anne-Marie Stretter, Michael Richard et Charles Rossett :

L'inconnu qui l'écoute a le visage blanc du vice-consul de Lahore. Charles Rossett

chasse l'image de la folie.

- Vous dormez debout?

Elle rit. Michael Richard la caresse ${ }^{7}$.

12 Sur le plan cognitif, la place que le texte accorde à l'espace n'intéresse pas seulement le processus de représentation; elle joue également un rôle dans la programmation du régime de lecture.

\section{Le cadre et la programmation du régime de lecture}

13 On ne lit pas toujours de la même façon. Comme l'ont montré les théoriciens de la lecture - et, en particulier, M. Picard ${ }^{8}-$, le choix fondamental est entre la lecture « distanciée » (qui, s'intéressant aux signifiés, prend le texte comme objet d'analyse) et la lecture «participative » (qui, soumise à l'illusion référentielle, privilégie le référent). Si l'on préfère les termes de B. Gervais l'alternative oppose la lecture «intensive » (ou 
«lecture-en-compréhension ») à la lecture «extensive» (ou «lecture-enprogression $»)^{9}$.

La plupart des chercheurs font dépendre le régime de lecture du libre choix du sujet lisant ${ }^{10}$. Il me paraît cependant que ce choix est largement programmé par le texte et, en particulier, par la façon dont l'espace est représenté. Lorsque le récit propose un espace détaillé et à forte valeur symbolique, il ralentit la progression et demande une lecture-en-compréhension. $\mathrm{Si}$, au contraire, il se contente de mentionner un espace stéréotypé, il appelle une compréhension fonctionnelle qui n'entrave pas la progression. On peut comparer de ce point de vue le traitement de l'espace dans deux textes aussi différents que Zazie dans le métro et Le Père Goriot. Dans le texte de Queneau, le narrateur se contente d'une simple mention des lieux traditionnels de la capitale (monuments, chaussée, boulevards, cafés) : cet espace stéréotypé, dont chaque composante correspond à un script de situation, permet une compréhension fonctionnelle qui favorise la lecture extensive :

A cause de la grève des funiculaires et des métrolleybus, il roulait dans les rues une quantité accrue de véhicules divers, cependant que, le long des trottoirs, des piétons ou des piétonnes fatigués ou impatients faisaient de l'auto-stop $(. . .)^{11}$.

En revanche, dans le roman de Balzac, la chambre du Père Goriot, en tant qu'espace détaillé (sa description s'étend sur près de quarante lignes), est dotée d'une forte valeur symbolique qui ralentit la progression et appelle une lecture en compréhension. Pour en donner une idée, on citera le passage suivant :

La fenêtre était sans rideaux; le papier de tenture collé sur les murailles s'en détachait en plusieurs endroits par l'effet de l'humidité, et se recroquevillait en laissant apercevoir le plâtre jauni par la fumée. Le bonhomme gisait sur un mauvais lit, n'avait qu'une maigre couverture et un couvre-pied ouaté fait avec les bons morceaux des vieilles robes de madame Vauquer. Le carreau était humide et plein de poussière ${ }^{12}$.

Ce bref extrait, en évoquant une chambre où rien n'échappe au dépérissement, où tout, jusqu'au moindre détail, est marqué par la décrépitude (les composants sont tous affectés de propriétés négatives) suggère une relation métonymique - très fréquente chez Balzac - entre l'habitat et l'habitant (le père Goriot est d'ailleurs inclus dans la liste des éléments qui composent la chambre). Les quelques verbes d'action, appliqués aux objets, leur donnent une sorte de vie inquiétante qui renforce par opposition la réification du personnage. La description, derrière sa dimension référentielle, demande donc à être interprétée sur le plan symbolique comme l'expression de l'existence misérable et pitoyable d'un personnage réduit à l'état de mort-vivant. Ainsi la question soulevée jadis par Genette - la description relève-t-elle de la mimesis (le "showing ») ou de la diegesis (le «telling») ? - n'est pas vraiment pertinente pour le lecteur : ce qui compte, pour lui, c'est de savoir quel type de lecture elle détermine: extensive ou intensive.

Qu'il s'agisse de diriger la représentation de l'action ou de programmer le régime de lecture, l'espace joue donc un rôle essentiel sur le plan cognitif. Mais les lieux revêtent également une importance non négligeable dans la dimension affective de la lecture. 


\section{Espace et identification : le rôle des lieux dans le processus affectif}

Sur le plan affectif, l'espace joue un rôle central dans la participation du lecteur au monde de la fiction. Ainsi, lorsque le texte choisit de programmer une lecture extensive, la description favorise la captation du lecteur en orientant son identification. Pour comprendre le processus ici en jeu, il convient de rappeler qu'on s'identifie, non à un personnage, mais à une situation ${ }^{13}$, c'est-à-dire à un réseau de relations. L'identification est, comme le rappelle Barthes, fondée sur une homologie des situations: «L'identification ne fait pas acception de psychologie; elle est une pure opération structurale: je suis celui qui a la même place que moi $\aleph^{14}$. Le parallélisme entre les situations proposées par le texte et la position du lecteur peut s'appréhender sur le plan du signifiant comme sur celui du signifié.

\section{L'homologie des situations : le plan du signifiant}

Sur le plan du signifiant, il y a homologie des situations lorsque le personnage est dans la même position que le lecteur par rapport à l'univers fictionnel. Ce parallélisme dépend essentiellement de l'espace mis en scène. L'alternative, pour le texte, est la suivante : soit introduire son personnage dans un espace inconnu, soit l'ancrer dans un décor familier.

Dans le premier cas, l'espace à découvrir se présente comme un vecteur d'identification très efficace. Comme le personnage arrivant dans un lieu inconnu, le lecteur pénètre lui aussi en nouveau dans l'espace du récit. Examinons, à titre d'exemples, les débuts de Vendredi ou les limbes du Pacifique de Tournier et du Château de Kafka. Après un bref prologue, le premier chapitre du roman de Tournier commence ainsi :

Une vague déferla, courut sur la grève humide et lécha les pieds de Robinson qui gisait face contre sable. A demi-inconscient encore, il se ramassa sur lui-même et rampa de quelque mètre vers la plage ${ }^{15}$.

\section{L'incipit du Château est le suivant :}

Il était tard lorsque K. arriva. Une neige épaisse couvrait le village. La colline était cachée par la brume et par la nuit, nul rayon de lumière n'indiquait le grand château. K. resta longtemps sur le pont de bois qui menait de la grand-route au village, les yeux levés vers ces hauteurs qui semblaient vides ${ }^{16}$.

2 Le lecteur, découvrant progressivement l'univers du texte comme le héros de $\mathrm{M}$. Tournier découvre son île et $\mathrm{K}$. l'espace inquiétant qui l'entoure, est spontanément enclin à identifier son regard à celui du personnage.

3 Dans le second cas, la reconnaissance d'un décor familier conduit le lecteur à retrouver immédiatement ses marques. Il s'identifiera tout naturellement à un personnage vivant dans les mêmes lieux que lui. C'est un procédé sur lequel jouent Butor dans $L a$ Modification (qui n'est pas familier avec l'espace du train ?) ou Jean-Philippe Toussaint dans La Salle de Bain (décor dont les composantes et accessoires sont connus de tous les lecteurs contemporains).

Nous dirons que, dans le premier cas (l'espace à découvrir), le texte construit une identification originale, alors que dans le second (l'espace familier), il joue sur une identification préexistant à la lecture. 


\section{L'homologie des situations : le plan du signifié} confronté à une scène qui, renvoyant à des modèles archétypaux ${ }^{17}$, a forcément été vécue par le lecteur. Dans la mesure où les fantasmes originels sont, pour la plupart, liés à des lieux, il est facile pour un texte de les réactiver en utilisant les motifs spatiaux: les cavernes, cercles et sous-terrains pourront suggérer «la vie intrautérine »; les portes, chambres et serrures " la scène originaire »; la nature vierge et sauvage «la mère archaïque ", etc. A titre d'exemples, on évoquera la transposition zolienne de la scène originaire où un personnage voit ce qu'il ne devrait pas (Nana surprise par Muffat dans les bras d'un autre homme, Jacques Lantier assistant, dans La Bête humaine, au meurtre du président Grandmorin) ${ }^{18}$ et le mythe de la robinsonnade (fantasme d'omnipotence et de maîtrise de l'espace qui renvoie, comme l'a montré Marthe Robert, au roman familial de l'enfant pré-oedipien ${ }^{19}$ ). Les contes jouent, bien sûr, de façon privilégiée, sur cette valeur fantasmatique de l'espace. M. Picard, dans une étude sur La Fée aux miettes de Nodier, a relevé la valeur exemplaire de l'opposition entre la Maison maternelle, "Maison primordiale d'où nous regrettons si souvent, nolens volens, d'être sortis ${ }^{20}$ et l'Auberge, lieu du voyage et de la confrontation avec l'altérité.

En programmant ainsi l'investissement affectif du lecteur, l'espace est toujours révélateur de l'intention qui anime le texte.

\section{Espace et intention : le rôle des lieux dans l'argumentation}

"Lire, comme le note Ph. Hamon, c'est non seulement "suivre" une information linéarisée, mais c'est également la hiérarchiser, c'est redistribuer des éléments disjoints et successifs sous forme d'échelles et de systèmes de valeurs à vocation unitaire et spécifique, c'est reconstruire du global à partir du local $»^{21}$. S'il y a bien, de la part du lecteur, le besoin de tirer du texte une leçon, la structuration de l'espace, ne serait-ce qu'en établissant des hiérarchies et des divisions, permet de pointer l'intention du récit.

Les valeurs qui sous-tendent la vision du narrateur passent en effet par une figuration propre à chaque texte. Nombre de récits proposent une structure spatiale explicite (haut/bas, surface/profondeur, ici/ailleurs, etc.) qui, investie sémantiquement, témoigne d'un système de valeurs. Il y a ainsi, dans Le Sous-sol de Dostoïevski, le monde $\mathrm{du}$ dessous (celui de l'affectif, des rancœurs et du ressassement) et le monde du dessus (celui de la "normalité ", du social et des apparences). Dans Madame Bovary, la grande ville et ses fastes (espace de la vie rêvée) s'oppose à la bourgade de province (espace désenchanté du quotidien). Dans La Recherche, le « côté de chez Swann » et le « côté de Guermantes" définissent deux pôles d'aimantation qui permettent de classer les personnages.

Les lieux étant surdéterminés, l'itinéraire d'un personnage est toujours à la fois parcours de l'espace et parcours de sens. Le déplacement se présente comme la manifestation figurative du désir ${ }^{22}$. Que l'on pense à Tiffauges, pris entre l'Allemagne et 
la France dans Le Roi des Aulnes, ou à Bardamu dans Le Voyage au bout de la nuit dont le parcours existentiel passe par l'Europe, l'Afrique et l'Amérique pour revenir à son point de départ. Sur un plan plus local, on évoquera les différents lieux scandant l'ascension sociale de Gil Blas ou, significativement, le refus du déplacement (manifestation de l'absence de désir ?) qui caractérise le personnage de Des Esseintes dans A Rebours :

A quoi bon bouger, quand on peut voyager si magnifiquement sur une chaise ?23

Si l'espace est ainsi lié à l'intention discursive qui imprègne tout récit, on comprend qu'il puisse jouer un rôle dans la portée symbolique du texte.

\section{Espace et signification : le rôle des lieux dans la symbolisation}

31 Ce que je voudrais montrer dans ce dernier temps de mon exposé, c'est que la dimension symbolique du texte apparaît comme indissociable de l'analyse de l'espace. $C^{\prime}$ 'est en effet lorsque des modèles sémantiques abstraits fondés sur des catégories très générales sont mis en relation avec des structures figuratives spatiales que le texte se donne à lire comme discours symbolique.

Pour Greimas, les structures sémantiques élémentaires d'un texte se ramènent essentiellement à deux modèles situés au niveau abstrait (profond et non-figuratif) ${ }^{24}$ :

\section{MODELE I \\ Univers individuel}

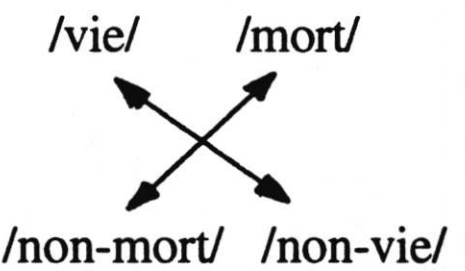

\section{MODELE II \\ Univers collectif}

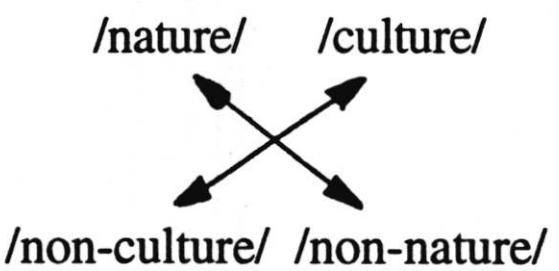

C'est lorsque l'un ou l'autre de ces carrés sémantiques est indexé sur les quatre éléments - feu, eau, air, terre - dont Bachelard a montré l'importance pour l'imaginaire ${ }^{25}$ que l'on peut dégager dans le texte une structure axiologique figurative (les éléments prenant valeur de symboles). Greimas a ainsi mis au jour dans Deux Amis de Maupassant un univers figuratif sociolectal (correspondant aux représentations collectives) issu du modèle II et un univers figuratif idiolectal issu du modèle I. Dans la nouvelle, la vie est figurée par le feu (le soleil), la mort par la terre (le Mont-Valérien), la non-mort par l'eau (la rivière) et la non-vie par l'air (le ciel). La réunion du soleil et de l'eau se présente donc comme euphorique, tandis que celle du ciel et du MontValérien est dysphorique. La première conjonction est du côté de l'affirmation vitale, la seconde du côté des forces de destruction. Le texte de Maupassant renvoie ainsi à des archétypes bibliques auxquels il fait subir des déformations individuelles. En effet, alors que dans l'imaginaire chrétien, le ciel, figurant la vie éternelle, est lié à la plénitude et à l'accomplissement, dans la nouvelle de Maupassant, il évoque également - et contradictoirement - la vacuité et l'absence de tout sens: «on aurait affaire ici, remarque Greimas, à une procédure assez curieuse qui consiste à exploiter des 
représentations chrétiennes sociolectales afin de dénier, de manière idiolectale, d'autres représentations chrétiennes; autrement dit, on assisterait à la production d'un mythe anti - et para - chrétien selon un modèle chrétien ${ }^{26}$. La référence évangélique, convoquée grâce à la sémantisation de l'espace, invite ainsi à lire le texte sur le plan mythique ; mais, une fois le contrat de lecture passé, c'est son propre mythe — « laïc » si l'on peut dire - que présente Maupassant.

F. Rastier propose d'élargir le champ de ces catégories sémantiques fondamentales qu'il nomme «dimensions»: «Les dimensions sont articulées entre elles par de grandes oppositions qui traversent les univers sémantiques, par exemple //animé// vs // inanimé//, //animal// vs //humain//,//animal// vs //végétal//27. Correspondant à la classe générique la plus générale, les "dimensions ", dont la valeur anthropologique semble avérée (voir, en particulier, les études de Lévi-Straus ${ }^{28}$ ), relèvent de l'archithématique, "partie de la thématique qui traite de la division des univers sémantiques en espaces valués $»^{29}$. Ces dimensions sont souvent liées à la configuration spatiale. Ainsi, chez Malraux, le surhumain (qui est symbolisé par l'élévation et la trouée) s'oppose à l'inhumain (qui est illustré par le sous-terrain, le grouillement, l'abîme et la fracture) comme le haut s'oppose au bas et l'ouvert au confiné. Examinons, de ce point de vue, deux courts passages de La Condition Humaine.

Voici le premier :

Cette vie des nuages animait l'obscurité, tantôt plus légère et tantôt intense, comme si d'immenses ombres fussent venues parfois approfondir la nuit ${ }^{30}$.

Le narrateur y insiste sur le mouvement et l'ouverture, motifs annonciateurs de l'insurrection victorieuse du lendemain.

Si l'on se réfère maintenant à l'extrait suivant :

La brume, nourrie par la fumée des navires, détruisait peu à peu au fond de l'avenue

les trottoirs pas encore vides ${ }^{31}$,

on remarque que l'accent est mis, au contraire, sur la fermeture (les fumées barrent verticalement le ciel) et la dissolution. Ce que révèle ici le paysage, c'est l'enfermement intérieur et les pulsions de mort du terroriste Tchen qui s'apprête à commettre un attentat.

Tout se passe comme si la signification la plus générale d'un texte (celle que l'on qualifie de "symbolique ») était toujours liée à la relation que propose le texte entre l'homme et l'espace - ce qui est, somme toute, logique, puisque les rapports de l'homme et du sens se sont d'abord manifestés dans la marque que ce dernier a imprimée à son environnement. Des textes comme Robinson ou L'île mystérieuse sont là pour nous rappeler cette vérité élémentaire ${ }^{32}$.

Il apparaît donc que les lieux jouent un rôle essentiel dans l'acte de lecture envisagé dans la diversité de ses dimensions. On peut constater que, significativement, les nouveaux romanciers, dans leur volonté de remettre en question les fondements du récit romanesque, n'ont pas touché à l'espace. La description est, au contraire, surévaluée, voire hypertrophiée, dans nombre de nouveaux romans (que l'on songe aux textes de Butor ou à ceux de Robbe-Grillet ${ }^{33}$ ). Un roman sans personnages est possible, un roman sans histoire peut-être, mais pas un roman sans espace. 


\section{NOTES}

1. «Pour une sémiotique de la lecture », Protée, 2-3, 1990, pp. 1-14.

2. Cf. Récits et actions, Longueuil, le Préambule, 1990.

3. Rappelons la définition générale du « script » donnée par B. Gervais (Récits et actions, op. cit., p. 165) comme «mode d'accomplissement standardisé, prévisible, qui n'a pas à être présenté dans sa totalité dans un discours ou un récit pour être reconnu par l'interlocuteur ou le lecteur ». Aller au restaurant, prendre le train, fonctionnent ainsi comme des scripts.

4. «Serait lisible, selon Ph. Hamon, quelque chose qui nous donnerait la sensation du déjà vu (ou déjà lu ou déjà dit, par le texte ou par l'extra-texte diffus de la culture) ; serait illisible un texte qui s'écarterait de ce déjà vu« («Un discours contraint » Poétique, 16, p. 120).

5. Voir, en particulier, le rendez-vous nocturne entre Emma et Rodolphe (Flaubert, Madame Bovary, Gallimard, coll. « Folio », 1979, pp. 119-120).

6. L'abus des scripts d'usage peut cependant avoir deux autres effets : 1 / attirer l'attention sur le discours lui-même plutôt que sur l'univers narratif, 2/conférer une espèce d'étrangeté à des actions stéréotypées (un geste banal - comme gommer un trait - décrit sur une dizaine de pages prend assez facilement une dimension symbolique). Cet effet très particulier de la surutilisation des scripts d'usage a été largement exploité par le Nouveau Roman, le faisant ainsi basculer de l'hyperréalisme vers l'étrange, voire le fantastique. Les textes de Robbe-Grillet en sont un bon exemple.

7. M. Duras, Le Vice-Consul, Paris, Gallimard, Coll. «L'Imaginaire », 1966, p. 197.

8. Cf. M. Picard, La Lecture comme jeu et Lire le temps, Paris, Minuit, 1986 et 1989.

9. Cf. B. Gervais, A L'Écoute de la lecture, Montréal, VLB, 1993. La lecture intensive est herméneutique et rétroactive, la lecture extensive heuristique et linéaire.

10. S'intéressant à la lecture concrète, ils font valoir que les réactions d'un lecteur réel ne tiennent pas forcément compte des exigences du texte. Voir, en particulier, J.-L. Dufays, Stéréotype et lecture, Liège, Mardaga, 1994.

11. R. Queneau, Zazie dans le métro, Paris, Gallimard, Coll. « Folio », 1959, p. 108.

12. Balzac, Le Père Goriot, Paris, Gallimard, Coll. « Folio », 1971, p. 177.

13. "On s'“identifie" non à un personnage, comme on le croit régulièrement, mais à un personnage en situation. Cette appropriation singulière à quoi pousse le besoin de lire conduit, quelle qu'en soit la base libidinale lointaine (...), à intégrer temporairement, comme pour les essayer, des situations, dont les héros ont seulement pour fonction de dessiner les contours » (M. Picard, La Lecture comme jeu, op. cit., pp. 93-94).

14. R. Barthes, Fragments d'un discours amoureux, Paris, Seuil, Coll. « Tel Quel », 1977, p. 153.

15. M. Tournier, Vendredi ou les limbes du Pacifique, Paris, Gallimard, Coll. « Folio », 1972, p. 15.

16. Kafka, Le Château, trad. franç., Paris, Gallimard, Coll. « Folio », 1938, p. 7.

17. Voir la définition des «fantasmes originaires » donnée par J. Laplanche et J.-B. Pontalis (Vocabulaire de la psychanalyse, Paris, P.U.F., 1967, p. 157) : «Structures fantasmatiques typiques (vie intra-utérine, scène originaire, castration, séduction) que la psychanalyse retrouve comme organisant la vie fantasmatique, quelles que soient les expériences personnelles des sujets ».

18. Cf. R. Ripoll, «Fascination et fatalité : le regard dans l'œuvre de Zola », in Les Cahiers naturalistes, 32, 1966.

19. Cf. M. Robert, Roman des origines et origines du roman, Paris, Gallimard, 1972.

20. M. Picard, La Fée aux miettes : loup y es-tu ?, Paris, PUF, Coll. « Le texte-rêve ", 1992, p. 61.

21. Ph. Hamon, Texte et idéologie, Paris, PUF., Coll. « Écriture », 1984, p. 54. 
22. "le déplacement, on le sait, s'interprète généralement, dans le cadre narratif, comme la manifestation figurative du désir, autrement dit, comme la forme narrative de la modalité du vouloir dont se trouve doté le sujet » (A.-J. Greimas, Du Sens II, Paris, Seuil, 1983, p. 146).

23. J.-K. Huysmans, A rebours, Paris, Garnier-Flammarion, 1978, p. 174.

24. A.J. Greimas, Maupassant - La sémiotique du texte, op. cit., p. 139.

25. Voir, entre autres, La Poétique de l'espace, Paris, P.U.F., 1957.

26. Cf. A. -J. Greimas, Maupassant- La sémiotique du texte, Paris, Seuil, 1976, p. 238.

27. F. Rastier, Sens et textualité, Paris, Hachette, 1989, p. 56.

28. Cf. Mythologiques, Paris, Plon, 1964-1971.

29. F. Rastier, Sens et textualité, op.cit., p.65.

30. Malraux, La Condition Humaine, Paris, Gallimard, Coll. « Folio », p. 23.

31. Ibid, p. 234.

32. Comme le note M. Picard à propos du roman de Jules Verne (La Lecture comme jeu, op. cit., p. 177), l'appropriation de l'espace par les personnages a quelque chose de forcené: «Une insistance d'abord curieuse, puis presque louche sur le mot "fouiller" donne en effet à cette "investigation" quelque chose de passionnel, d'obstiné, presque de sauvage ».

33. C'est ce primat de la description qui explique qu'on ait pu qualifier le Nouveau Roman de « littérature objective » ou d'« école du regard». En mettant l'accent sur l'espace, il s'agissait, pour les nouveaux romanciers, de débarrasser la vision du monde de toute dimension anthropocentriste en le réduisant à sa matérialité, à un "être-là » indifférent à l'homme. L'importance accordée à l'espace était la contrepartie naturelle de la méfiance envers le personnage. Comme l'écrivait Bernard Pingaud, «le monde extérieur gagne en importance ce que l'homme a perdu » (Esprit, juillet-août 1958).

\section{AUTEUR \\ VINCENT JOUVE}

Université de Reims 